\title{
Significance of Rate of Work Hardening in Tempered Martensite Embrittlement
}

\section{J. Pietikainen}

Laboratory of Physical Metallurgy and Material Science, Helsinki University of Technology, 02150 Espoo, Finland

\begin{abstract}
The main explanations for tempered martensite embrittlement are based on the effects of impurities and cementite precipitation on the prior austenite grain boundaries. There are some studies where the rate of work hardening is proposed as a potential reason for the brittleness.One steel was studied by means of a specially developed precision torsional testing device. The test steel had a high $\mathrm{Si}$ and $\mathrm{Ni}$ content so $\varepsilon$ carbide and $\mathrm{Fe}_{3} \mathrm{C}$ appear in quite different tempering temperature ranges. The $\mathrm{M}_{\mathrm{S}}$ temperature is low enough so that self tempering does not occur.With the testing device it was possible to obtain the true stress - true strain curves to very high deformations. The minimum toughness was always associated with the minimum of rate of work hardening.The change of deformed steel volume before the loss of mechanical stability is proposed as at least one reason for tempered martensite embrittlement.The reasons for the minimum of the rate of work hardening are considered.
\end{abstract}

\section{INTRODUCTION}

Tempered martensite embrittlement (TME) is well known and has been much studied. Several explanations for embbrittlement have been presented. Perhaps the first explanation for TME was given by Grossman [1]. He thought that it was caused by the decomposition of retained austenite in one way or another. The hypothesis of mechanical instability was presented by Read et al. [2]. Quite popular explanations for TME have been the effects of impurities and $\mathrm{Fe}_{3} \mathrm{C}$ precipitation along with the impurity weakened prior austenite grain bountaries [3,4]. Zia-Ebrahimi and Krauss reviewed several mechanisms presented in the literature [5], and based on tests and observations they concluded that phosphorus is not the reason for TME. They mentioned that "all modes of TME, ductile or brittle, arise from the combination of carbide formation and changes in strain- hardening behaviour produced by tempering".

The Charpy test is the most frequently used for the observation of TME even though the torsion impact test of Luerssen and Green [6] gave excellent results even in the case of high carbon steels. The torsion test method was further improved by Pietikäinen [7].

The present paper is an attempt to further elaborate the earlier mechanical instability hypothesis $[2,7]$.

\section{MATERIALS AND EXPERIMENTAL PROCEDURES}

The chemical composition of the steel used in this study is given in Table 1 .

Table 1.Chemical composition of the steel investigated in wt. \%

\begin{tabular}{ccccccccccc}
\hline $\mathrm{C}$ & $\mathrm{Ni}$ & $\mathrm{Si}$ & $\mathrm{Mn}$ & $\mathrm{P}$ & $\mathrm{S}$ & $\mathrm{Cr}$ & $\mathrm{Mo}$ & $\mathrm{Cu}$ & $\mathrm{Sn}$ & $\mathrm{B}$ \\
.34 & 20.1 & 1.41 & .42 & .012 & .018 & .07 & .06 & .18 & .02 & .0004
\end{tabular}

The steel was manufactured by an industrial arc-melting procedure. Owing to the raw materials and smelting procedure, the steel contained a fair amount of impurities.

The composition of the steel was selected so that the 
$\mathrm{M}_{\mathrm{s}}$ temperature would be below room temperature but after subzero cooling the amount of retained austenite would be about $10 \%$ and $\varepsilon$-carbide and $\mathrm{Fe}_{3} \mathrm{C}$ formation would occur over separate tempering temperature ranges. The carbon content selected was low enough so that the fracture always occurred by shearing in the torsion tests.

Austenitizing temperature conditions were $\mathrm{T}_{\gamma}=1200{ }^{\circ} \mathrm{C}$ and time $t_{\gamma}=30 \mathrm{~min}$. The treatment gave an austenite grain size of 3 (ASTM) and an $M_{S}$ temperature of about $+15^{\circ} \mathrm{C}$. Because of the large austenite grain size, the $M_{S}$ temperature was a little higher than expected. The specimens were encapsulated in quartz during austenitizing and were quenched into warm brine in which the capsule was broken. Then the specimens were quenched into liquid nitrogen. The tempering time was $1 \mathrm{~h}$ at the selected temperatures of $70,190,310$ and $430^{\circ} \mathrm{C}$.

Dilatometric, $\mathrm{X}$-ray, surface replica and thin foil electron microscope examinations were made to identify the reactions and carbides formed. For identification, the carbide separation was very useful. The methods are described elsewhere [7].

Mechanical tests were made with a precision torsional testing devise. Its main design is described elsewhere [7]. The testing device was modified by re - building the test head so that the testing temperatures were easily set. A TV camera, AV microcomputer and VCR were connected so that all elements for a true stress- true strain diagram were automatically registered.

The test specimen is shown in Fig.1. After the heat treatment, the specimens were polished and on their neck part black lines were etched parallel with the axes of the specimen using light sensitive lacquer techniques and Se-acid. During the test the tubular specimen was filled with a TiN coated hardened steel pin lubricated with MoS grease. The tests presented in this paper were conducted at $25^{\circ} \mathrm{C}$.

\section{RESULTS}

Some $\tau$ - $\operatorname{tg} \gamma$ curves at medium speeds of rotation are shown in Fig.2. As we can see, tempering temperatures have a big effect on the curves. In the case of a low tempering temperature, the rate of work hardening is high. With increasing tempering temperature the rate of work hardening decreases but increases again at the highest temperatures.Increases in speed of rotation decrease the rate of work hardening. In the case of $\mathrm{T}_{\mathrm{t}}=190$ and $310^{\circ} \mathrm{C}$ the curves have kinks. These kinks formed with a deformation band forming mechanism. For examle, in the case $T_{t}=310^{\circ} \mathrm{C}, \mathrm{n}=4.231 / \mathrm{min}$ a highly deformed band was formed at (a) in Fig. 1 and (a-a') in Fig.2. When the band spread from (a) to (b) in Fig.1 the stress increased ( $a^{\prime}$ to $b$ ) in Fig. 2 . Then at stress stage (b) in Fig. 2 a new deformation band (b- $b^{\prime}$ ) formed and it spread from (b) to (c) in Fig.1 and the stress stage increased from (b) to (c) in Fig.2. In this case, four bands formed with the last one causing the fracture. The forming of a band is accompanied by a definite sound, quite audible in the testing machine.

In Fig.3. we have the $\tau-\operatorname{tg} \gamma$ curves obtained at high testing speeds. Compared with the Fig. 2 curves the curves have been shifted down and their initial ascent has steepened. It is to be particularly noted that the curves decend after a certain deformation, which implies that work softening is occuring in the test specimens.

In Fig. 4 we have a total twist $\varphi_{\mathrm{ft}}$ as a function of $\log$ test speed $(\log \mathrm{n})$ in the case of different tempering temperatures. The total twist $\varphi_{\mathrm{ft}}$ indicates how many degrees the specimen twisted before breaking. At the tempering temperature $T_{t}=70^{\circ} \mathrm{C}$ the total twist curve jumps upward between the speed of $n=0.1$ and 1 and then declines with increasing speed. In the cases of $T_{t}=190$ and $310^{\circ} \mathrm{C}$ the $\varphi_{\mathrm{ft}}$-curves first increase and then fall. After the fall they continue to have a decreasing trend. The $T_{t}=430{ }^{\circ} \mathrm{C}$ curve declines uniformly and it has no jumps.

Local shear strain $\operatorname{tg} \gamma_{1}$ measured in the fractured specimen as a function of the bosition on the neck portion of the specimen are shown in Fig.5, one below another with increasing test speed in the case of $T_{t}=190$, 310 and $430^{\circ} \mathrm{C}$. The jumps seen in Fig. 4 can also be seen in Fig. 5 in the cases of $\mathrm{T}_{\mathrm{t}}=190$ and $310^{\circ} \mathrm{C}$. In the case of $\mathrm{T}_{\mathrm{t}}=430^{\circ} \mathrm{C}$ the deformed volume decreases quite evenly with increasing test speed.

Fig. 6. gives the total twist $\varphi_{\mathrm{ft}}$, the energy consumed in fracturing the test specimen $W$, the maximum shear stress $\tau_{\max }$ derived from the $\tau$-tgy curves and the hardness of the specimen $\mathrm{H}_{\mathrm{V}}$ as a function of tempering 


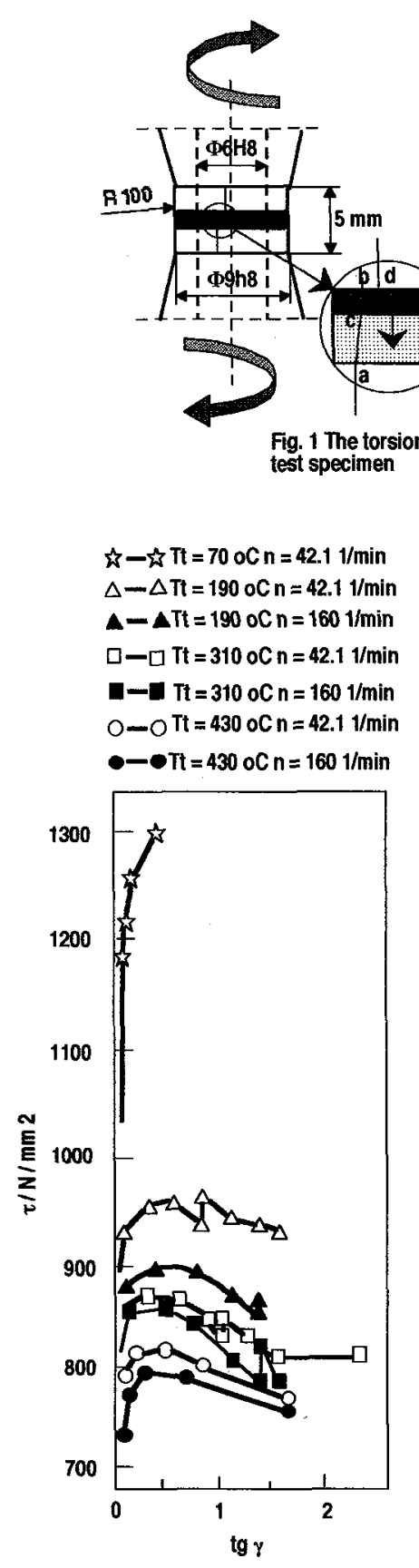

Fig.3. $\tau$-tgy curves at high speed of rotation

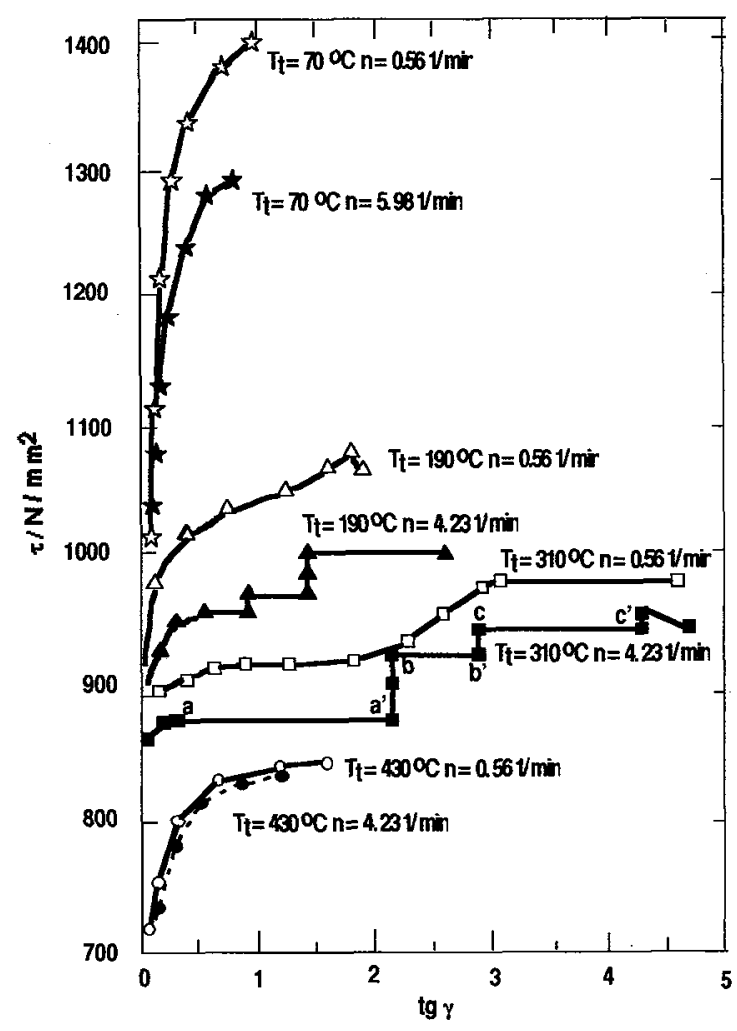

Fig. 2. $\tau-\operatorname{tg} \gamma$ curves at medium speed of rotation

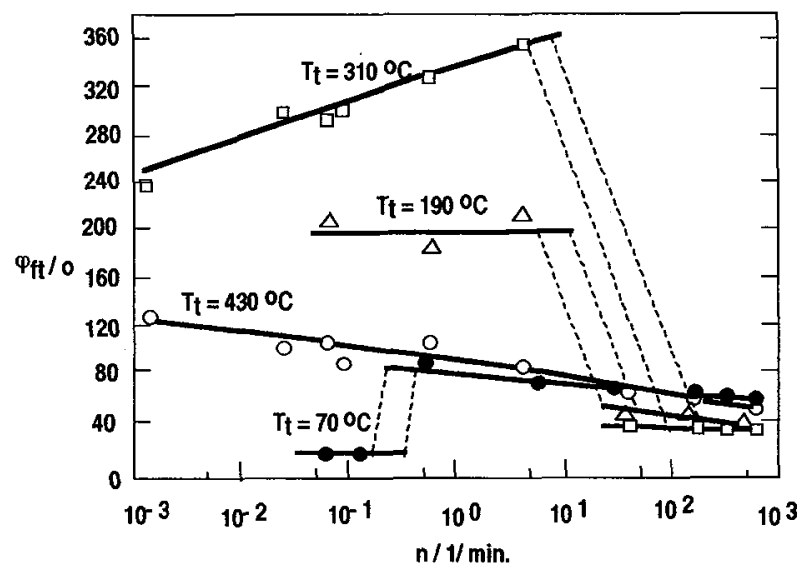

Fig. 4. Total twist $\varphi f t$ as a function of $\log n$ in the case of different tempering temperatures $T_{t}$ 


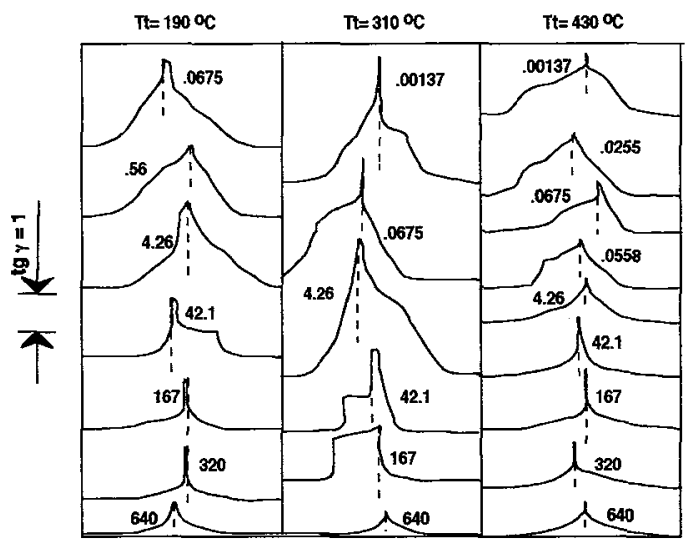

$\rightarrow<1=1 \mathrm{~mm}$

Fig. 5. Local shear stain - length curves for various tempering temperatures Tt and speed of rotation
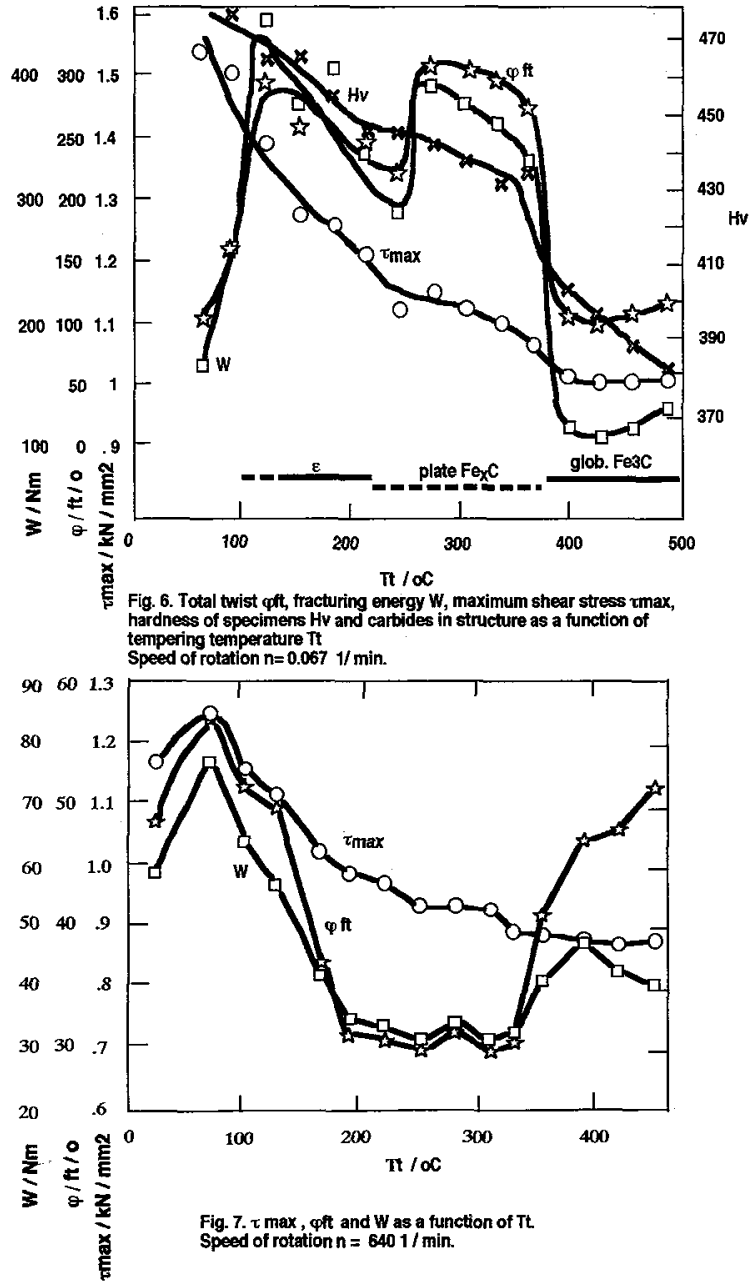
temperature $T_{t}$. The testing speed was $n=.0671 / \mathrm{min}$. In Fig. 6 we also have the results of $x$-ray and microscope analyses of carbides present in the structure after tempering. As we can see, the $\mathrm{H}_{\mathrm{V}}$ curve has a decreasing tendency with increasing $T_{t}$. There is a distinct turn upwards between $T_{t}=c .200-380{ }^{\circ} \mathrm{C}$. The $\tau_{\max }$ curve has the same tendency and turns upwards as does the $\mathrm{H}_{\mathrm{V}}$ curve. The turn upwards occurs in the same $T_{t}$ areas where we have plate $\mathrm{Fe}_{\mathrm{x}} \mathrm{C}$ carbides in the structure. In the case of the $\varepsilon$ - carbide and globular $\mathrm{Fe}_{3} \mathrm{C}$, the $\mathrm{x}$-ray lines fit quite well with the standard values but in the case $\mathrm{Fe}_{\mathrm{X}} \mathrm{C}$ they did not. We think that $\mathrm{Fe}_{\mathrm{X}} \mathrm{C}$ is the coherent alloyed $\mathrm{Fe}_{3} \mathrm{C}$ but this is not certain. In the $\varphi_{\mathrm{ft}}$ and $\mathrm{W}$ curves we have two maxima. The first in the $\varepsilon$ - carbide region and the second in the $\mathrm{Fe}_{\mathrm{x}} \mathrm{C}$ region. There is a valley between these $\varepsilon$ and $\mathrm{Fe}_{\mathrm{x}} \mathrm{C}$ regions. The curves fall when the globular $\mathrm{Fe}_{3} \mathrm{C}$ appears in the structure.

In Fig.7. we have $\tau_{\max }, \varphi_{\mathrm{fr}}$ and $\mathrm{W}$ as a function of $T_{t}$ in the case of the testing speed $n=6401 / \mathrm{min}$. The curves have a maximum at low $T_{\mathrm{t}}$. Then the $\tau_{\max }$ curve decreases. The $\varphi_{\mathrm{ft}}$ and $\mathrm{W}$ curves have a deep valley in the $\mathrm{Fe}_{\mathrm{X}} \mathrm{C}$ area. The $\varphi_{\mathrm{ft}}$ and $\mathrm{W}$ curves are at a lower level in the case of Fig. 7 than in the case of Fig.6.

Surface replica and thin foil electron microscopy of the broken specimens showed that on the $\varepsilon$-carbide and globular $\mathrm{Fe}_{3} \mathrm{C}$ areas, sliplines were complicated and often crossed. In the $\mathrm{Fe}_{\mathrm{X}} \mathrm{C}$ area the deformation marks on the surfaces of the specimens were terrace-like. In the fine structure the carbide plates in the band areas were evenly orientated, the habit planes in the direction of the deformation in the matrix, and in the band boundaries, bent i.e.only partly re-oriented.There were also very local bands in the microstructure where the carbite plates were re-oriented in a narrow strip .

\section{DISCUSSION}

In the following discussion, the experimental results are examined from the points of view of the effects of the testing speed and rate of work hardening and the reason for the low rate of work hardening and band formation.

As we can see in Fig. 4 and by comparing Figures 6 and 7 the increase in the test speed dramatically decreases $\varphi_{\mathrm{ft}}$ and $W$. It happens especially in the $T_{t}$ area where the rate of work hardening is low. Simple adiabatic calculations indicate that the deformation energy increases the temperature of test specimens locally when the test speed is high enough and is the reason that the $\tau$-tg $\gamma$ curves turn downwards in Fig.3 and the deformation in the test specimen is localised as we can see in Fig.5. The spreading of deformation is hindered by the local softening.

Martensitic transformation causes quite a dense dislocation structure in steel and we can think of this dislocation structure as very stable in the case where the carbon content in the solid solution and/or in clusters is high. In the case of $\varepsilon$ - carbide we have quite a lot of carbon in solid solution, but in the case of $\mathrm{Fe}_{\mathrm{x}} \mathrm{C}$ the carbon content in solid solution may be low. It is likely that the dislocation structure becomes mechanically unstable and it is proposed that dislocations can move into new positions without or with very low work hardening. When enough carbon is in solid solution and when the deformation speed is low enough, the locking of dislocations can occur and contribute to band formation.

\section{CONCLUSIONS}

A C- Ni- Si- steel tempered at different tempering temperatures was studied by torsional testing with different testing speeds and analysed by metallographic and X-ray methods. It was observed that in the tempered martensite embrittlement area

- an increase in testing speed dramatically decreased the ductility of the steel tested. With low testing speed, the steel is very ductile. The deformation occurs by formation of bands and the rate of work hardening is very low. At higher testing speeds the deformation localizes. It was calculated that the reason for this appears to be the work of deformation which increases the local temperature and causes work softening.The change of deformed steel volume before the loss of mechanical stability is proposed as at least one reason for tempered martensite embrittlement.

- X-ray and microscopy studies indicated that in the embrittlement areas there were, in the microstruture, plate-like carbides which were called $\mathrm{Fe}_{\mathrm{X}} \mathrm{C}$ because the $\mathrm{X}$ - ray lines did not fit with the known carbides. In deformation these carbide plates turned their plate directions to the macro and micro band directions.

-it was thought that the reason for the low rate of work hardening and therefore one reason for tempered 
martensite embrittlement may be the metastability of the dislocation structure created in the martensitic transformation. The mechanical metastability of the dislocation structure is caused by the depletion of the ordered carbon- iron solid solution.

\section{Acknowledgments}

I wish to thank Prof.V. Lindroos and his staff for their help and kindness. I also wish to thank Imatra Steel Co., Högfors TTT Co., the Academy of Finland and Helsinki Univ. of Technology (HUT) for economic support. My warm thanks are due to the staff of the Laboratory of Production Engineering of HUT for help in the rebuilding of the testing machine. Last but not least, my thanks to Mr.Harvey Benson who corrected the English text.

\section{References}

[1] Grossman M.A.,Iron Age 114 (1924) July 3891

[2] Read T.A. et al.,Proc.Seminar on the Fract. of Metals. Ed. Johanson F. et al. Cleveland, ASM (1948) 228.

[3] Briant C.L. and Banerji S.K., Intern.Met.Rev. 4 (1978) 164-199, Rev.no.232.

[4] Briant C.L. and Banerji S.K., Met. Trans. 10A (1979) 1729-1737.

[5] Zia-Ebrahimi F. and Krauss G., Acta metall. 10 (1984) 1767-1777.

[6] Luerssen G.V. and Green O.V., Proc.ASTM 33 Part II (1933) 315.

[7] Pietikäinen J., Acta Polytech.Scand. Ch 47.(1966). 